\title{
Designing Intrinsic Topological Insulators in Two-Dimensional Metal-Organic Frameworks
}

Tianqi Deng ${ }^{1}$, Wen Shi ${ }^{1,2}$, Zicong Marvin Wong ${ }^{1}$, Gang Wu ${ }^{1 *}$, Xiaoping Yang ${ }^{3}$, Jin-Cheng Zheng ${ }^{4,5}$, Hui Pan ${ }^{6}$ and Shuo-Wang Yang ${ }^{1 *}$

${ }^{1}$ Institute of High Performance Computing, Agency for Science, Technology and Research, 1 Fusionopolis Way, \#16-16 Connexis, Singapore 138632, Singapore

${ }^{2}$ School of Chemistry, Sun Yat-sen University, 510275, Guangzhou, China

${ }^{3}$ Anhui Key Laboratory of Condensed Matter Physics at Extreme Conditions, High Magnetic

Field Laboratory, HFIPS, Anhui, Chinese Academy of Sciences, Hefei 230031, China

${ }^{4}$ Department of Physics, Xiamen University, Xiamen, 361005, China

${ }^{5}$ Department of Physics, Xiamen University Malaysia, Sepang, 43900, Selangor, Malaysia

${ }^{6}$ Institute of Applied Physics and Materials Engineering, University of Macau, Taipa, Macao, SAR, 999078 China

Table S1 The energy gap between $E_{2 u}$ and $B_{1 g}$ crystal orbitals $\left(\Delta E_{E_{2 u}-B_{1 g}}\right)$, between $E^{\prime \prime}$ and $A_{2}^{\prime \prime}$ linker orbitals $\left(\Delta E_{E^{\prime \prime}}-A_{2}^{\prime \prime}\right)$, and tight-binding parameters for single-orbital $\pi$-d honeycomb-kagome lattice, with $t_{\pi d}$ and $\Delta E_{d-A_{2}^{\prime \prime}}$ being hopping term and the onsite energy difference between linker $A_{2}^{\prime \prime}$ and metal $d$ orbital.

\begin{tabular}{|c|c|c|c|c|}
\hline & $\boldsymbol{\Delta}_{\boldsymbol{E}_{\boldsymbol{2} \boldsymbol{u}}-\boldsymbol{B}_{\boldsymbol{1} \boldsymbol{g}}}(\mathbf{e V})$ & $\boldsymbol{\Delta} \boldsymbol{E}_{\boldsymbol{E}^{\prime \prime}-\boldsymbol{A}_{\mathbf{2}}^{\prime \prime}}(\mathbf{e V})$ & $\boldsymbol{t}_{\boldsymbol{\pi} \boldsymbol{d}}(\mathbf{e V})$ & $\boldsymbol{\Delta} \boldsymbol{E}_{\boldsymbol{d}-\boldsymbol{A}_{\boldsymbol{2}}^{\prime \prime}}(\mathbf{e V})$ \\
\hline Ni-BHSe & -0.87 & 1.16 & 1.03 & -1.64 \\
\hline Ni-BHT & -0.74 & 1.36 & 1.08 & -1.86 \\
\hline Ni-HIB & 0.39 & 1.93 & 1.01 & -2.27 \\
\hline Ni-TABTT & -0.29 & 1.62 & 1.05 & -2.14 \\
\hline Pd-BHSe & -0.57 & 1.16 & 1.06 & -2.48 \\
\hline Pd-BHT & -0.41 & 1.36 & 1.11 & -2.66 \\
\hline Pd-HIB & 0.57 & 1.93 & 1.04 & -2.98 \\
\hline Pd-TABTT & 0.08 & 1.62 & 1.06 & -2.84 \\
\hline Pt-BHSe & -1.10 & 1.16 & 1.21 & -2.53 \\
\hline Pt-BHT & -1.04 & 1.36 & 1.29 & -2.73 \\
\hline Pt-HIB & -0.56 & 1.93 & 1.34 & -3.01 \\
\hline Pt-TABTT & -0.87 & 1.62 & 1.31 & -2.93 \\
\hline
\end{tabular}




\section{Supplementary Figures}

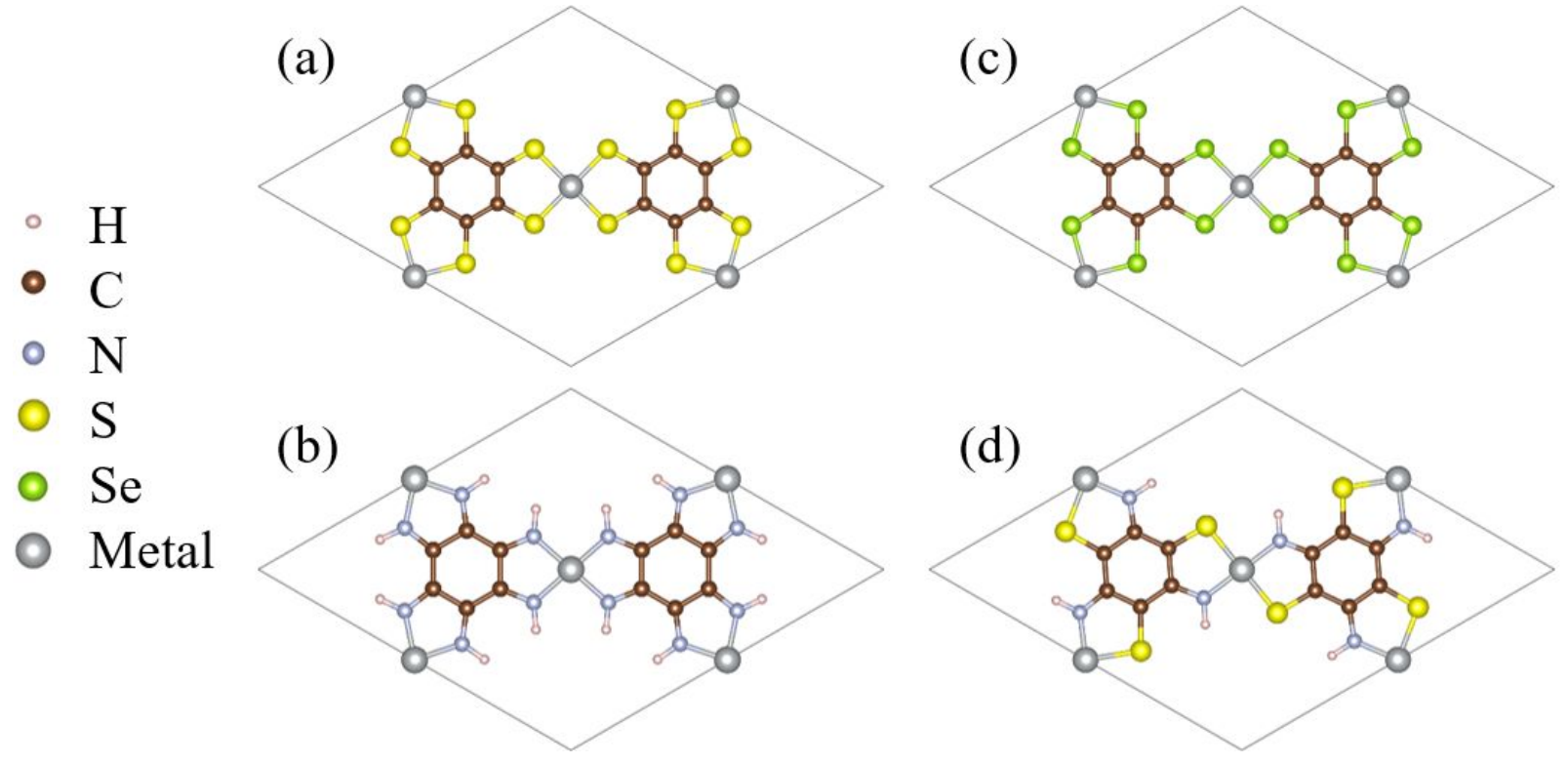

Figure S1 The general crystal structure of (a) M-BHT, (b) M-HIB, (c) M-BHSe, and (d) MTABTT, with metal $\mathrm{M}=\mathrm{Ni}, \mathrm{Pd}$, Pt.
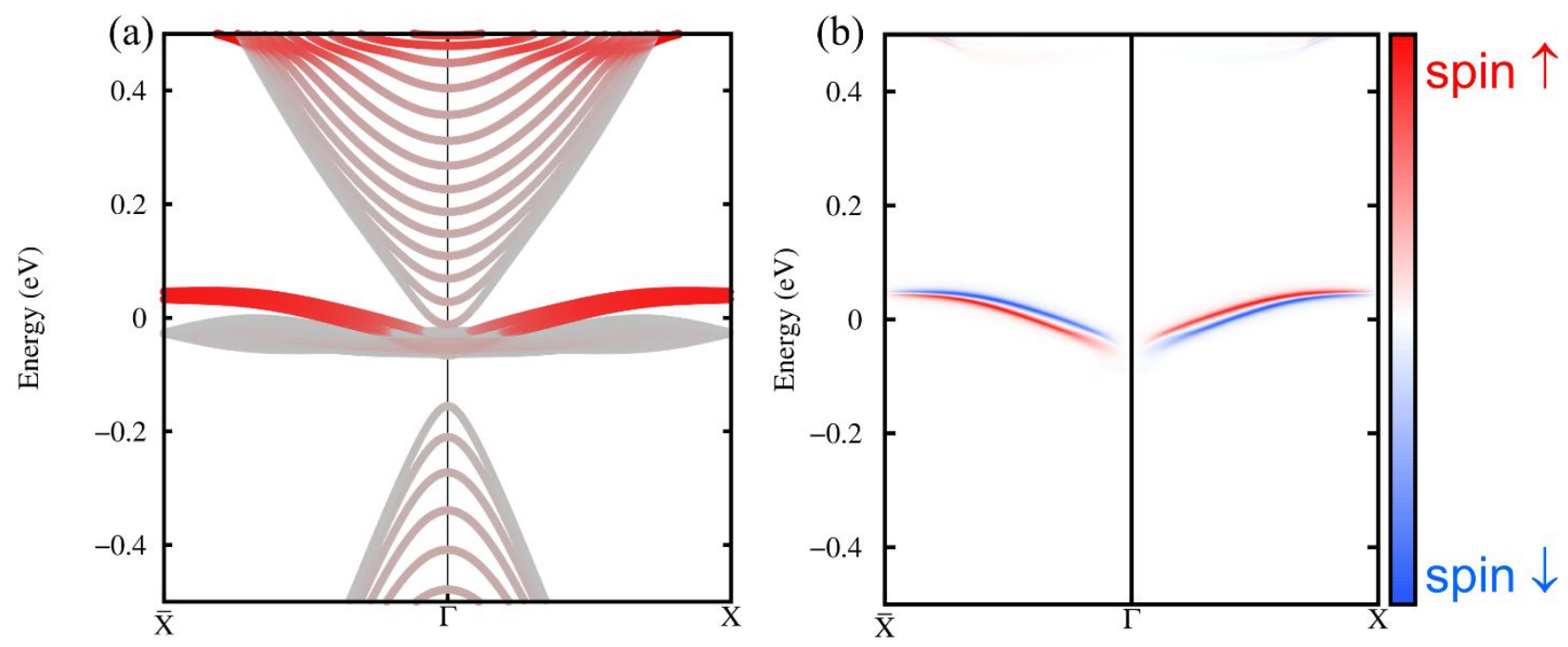

Figure S2(a) The band structure of a Pd-TABTT ribbon, where the redness corresponds to the edge contribution. (b) The spin polarization of the edge spectrum, defined as $A^{\uparrow}(\mathbf{k}, E)-A^{\downarrow}(\mathbf{k}, E)$ where $A^{\uparrow / \downarrow}(\mathbf{k}, E)$ are the edge spectral function contributions from the two spinors. 


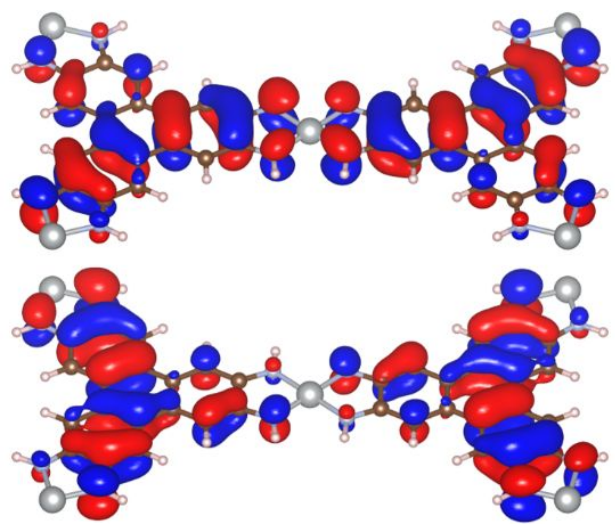

Figure S3 The non-bonding $\mathrm{E}_{2 \mathrm{u}}$ states in Ni-HITP.

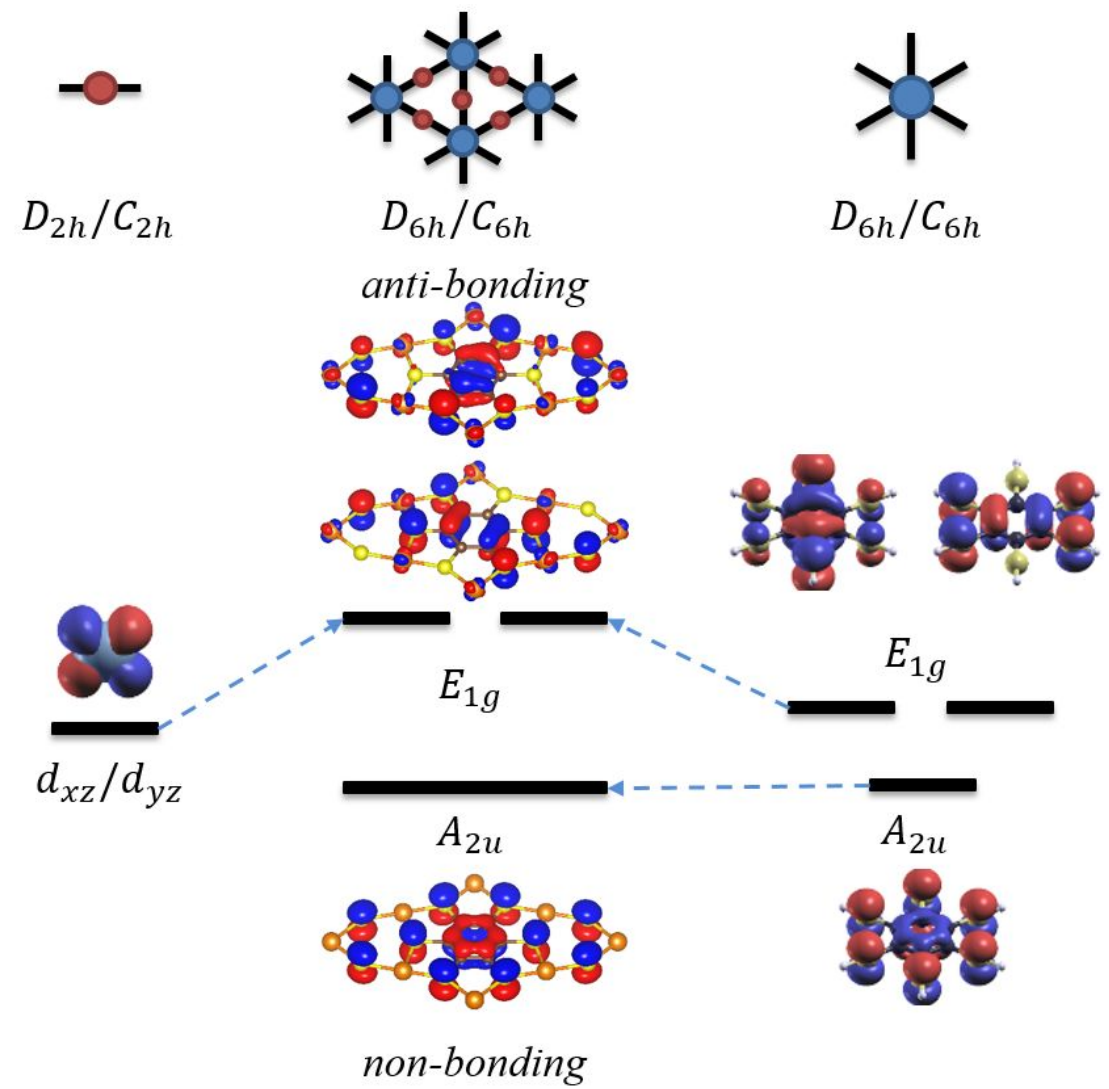

Figure $\mathbf{S} 4$ Orbitals diagram of $\mathrm{Cu}_{3}(\mathrm{BHT})$. As a result of different lattice geometry and topology, the linker orbital symmetry changes and the selection rule also becomes different from Ni-BHT. 


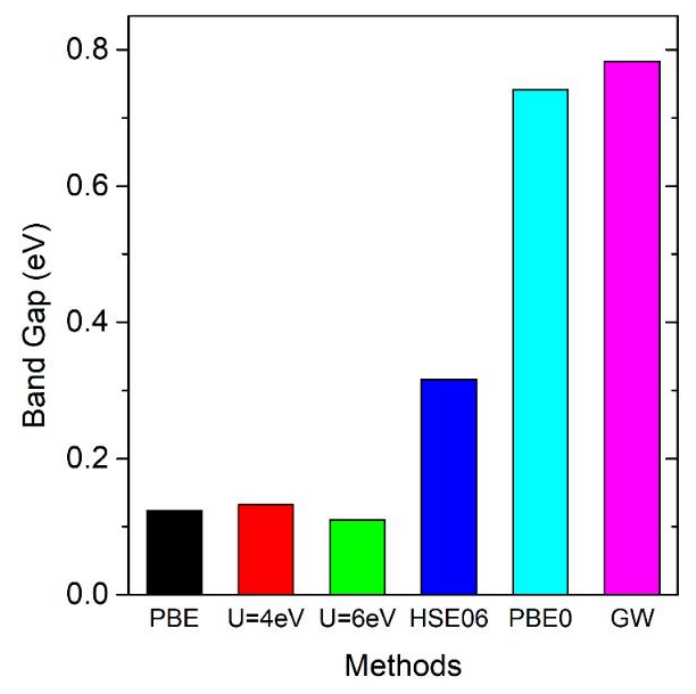

Figure S5 The band gaps of Ni-BHT computed using different methods.
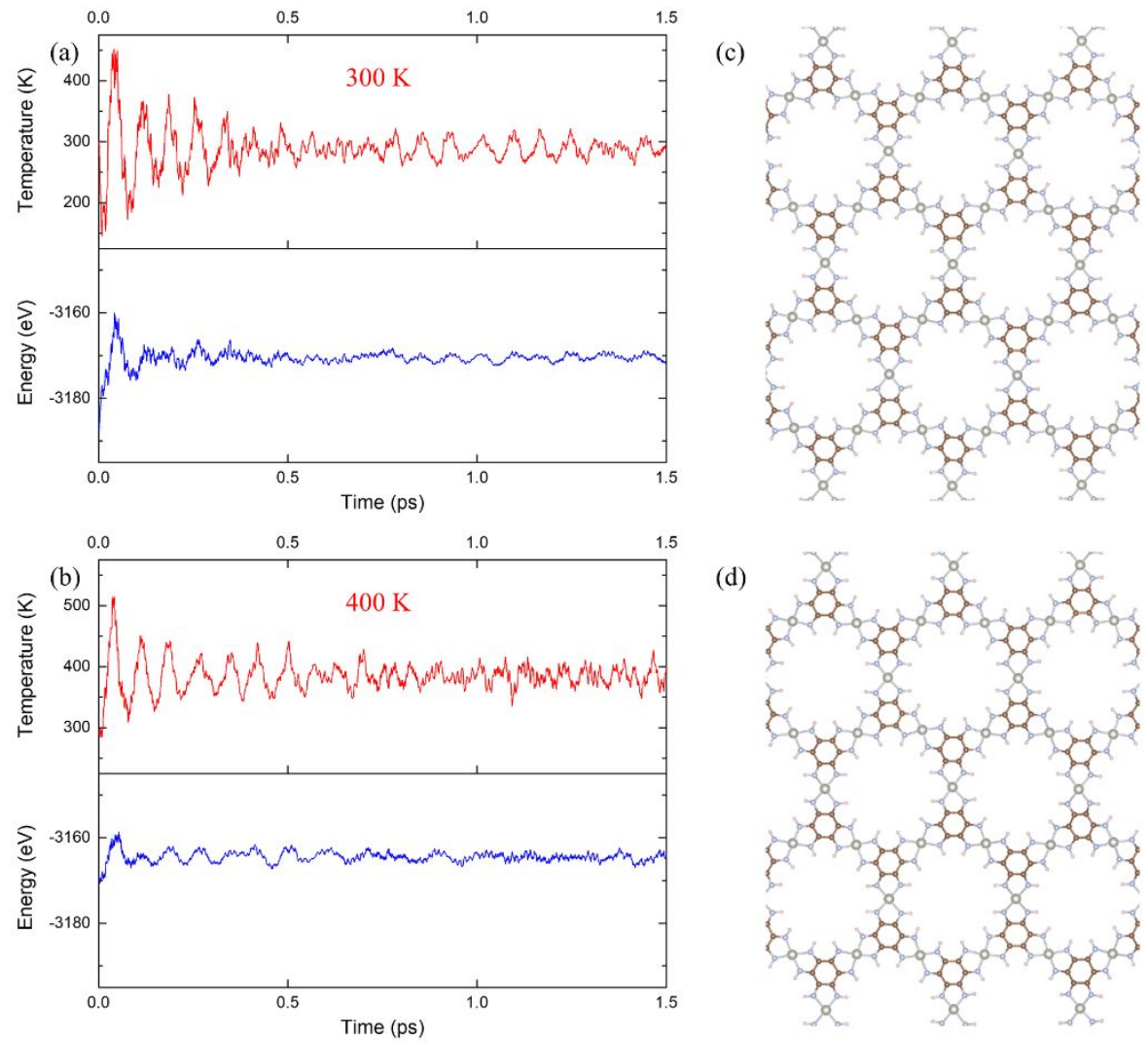

Figure S6 The $a b$ initio molecular dynamics (AIMD) simulation results. The simulated Pd-HIB system temperature and energy in NVT ensemble at (a) $300 \mathrm{~K}$ and (b) $400 \mathrm{~K}$. The Pd-HIB structure after AIMD simulations at (c) $300 \mathrm{~K}$ and (d) $400 \mathrm{~K}$. 


\section{Non-bonding states in related metal-organic materials}

The symmetry-protected non-bonding $\mathrm{E}_{2 \mathrm{u}}$ states are generally present in all MOFs studied in this work, and relate to many of their exotic electronic properties. One would naturally wonder whether analogous electronic states exist in related materials including two-dimensional and linear coordination systems with planar backbones. A triphenylene-based MOF with similar geometry, Ni-HITP (HITP stands for 2,3,6,7,10,11-hexaiminotriphenylene) which was also reported with high conductivity ${ }^{1}$ and mobility ${ }^{2,3}$, shows a significantly larger distance between metal centers as compared to the benzene-based MOFs studied in this work. However, the more fundamental symmetry relations among lattice, metal sites, and ligands remain unchanged. Therefore, similar delocalized non-bonding $E_{2 u}$ states are expected near Fermi level. And this is indeed confirmed in Ni-HITP, as shown in Figure S2. Moreover, as the ligand size increases from benzene to triphenylene derivatives, the molecular level spacing should decrease, resulting in overall smaller $\Delta E_{E^{\prime \prime}}-A_{2}^{\prime \prime}$. This may explain the observation in N-containing triphenylenebased MOFs where the $B_{1 \mathrm{~g}}$ state is much higher than $\mathrm{E}_{2 \mathrm{u}}$ states, making it difficult to realize intrinsic quantum spin hall insulator ${ }^{4,5}$.

On the other hand, $\mathrm{Cu}_{3}(\mathrm{BHT})$, which is a metallic and superconducting MOF possessing a different metal-to-ligand ratio ${ }^{6-8}$, has the same $\mathrm{D}_{6 \mathrm{~h}}$ crystal point group. However, while the local environment around $\mathrm{Cu}$ atom is similar to that of $\mathrm{Ni}$ in $\mathrm{Ni}-\mathrm{BHT}$, the BHT ligand is coordinated with six $\mathrm{Cu}$ atoms instead of three, which means that the $\mathrm{BHT}$ ligand in $\mathrm{Cu}_{3}(\mathrm{BHT})$ has a six-fold rotational symmetry just like benzene's $\mathrm{D}_{6 \mathrm{~h}}$ point group. Therefore, the ligand $\pi$ orbitals should be classified with the $A_{2 u}$ and $E_{1 g}$ representations in $D_{6 h}$ group instead of the $A_{2}^{\prime \prime}$ and $E^{\prime \prime}$ in $D_{3 h}$ group. The selection rule thus completely changed where the doubly degenerate $\mathrm{E}_{1 \mathrm{~g}}$ orbitals can hybrid with $\mathrm{Cu} d$ orbital while the $\mathrm{A}_{2 \mathrm{u}}$ orbital cannot, as illustrated in Figure $\mathrm{S} 3$. This produces the highly dispersive hole pocket in $\mathrm{Cu}_{3}(\mathrm{BHT})$ which is important for its metallic and superconducting property, while the non-bonding $A_{2 u}$ states are fully occupied and far away from Fermi level. In linear $\mathrm{d}^{8}$ coordination polymers with planar backbones, non-bonding states without involving metal $d$ orbitals can also be found near Fermi level and showed very small deformation potential constants, which were connected to their excellent potential in thermoelectric applications ${ }^{9,10}$. A similar connection between non-bonding states and symmetries can also be established, for example, by considering the $\mathrm{D}_{2 \mathrm{~h}}$ point group where ligand $\pi$ orbitals 
could realize $A_{u}, B_{1 u}, B_{2 g}$, and $B_{3 g}$ representations. Among these, only $B_{2 g}$ and $B_{3 g}$ representations are compatible with metal $d$ orbitals, thus forbidding hybridization between $d$ orbitals and $\mathrm{A}_{\mathrm{u}} / \mathrm{B}_{1 \mathrm{u}} \pi$ orbitals. The general presence of non-bonding orbitals in these materials also emphasizes the important role of coupling between non-bonding atoms' $\pi$ electrons. In our previous works, we have found that such couplings are responsible for many interesting phenomena in organic electronic materials, including metallization of 2D MOFs ${ }^{11}$, self-doping in coordination polymers ${ }^{12}$, and strong intermolecular coupling in molecular crystals ${ }^{13}$. We expect such non-bonding couplings to offer additional dimensions for materials engineering and design that enable further advances in organic electronics.

\section{Tight-binding model}

As discussed in the main text, the topological feature of MOFs studied here are determined by the $\Gamma$-point energy levels $E_{2 u}$ and $B_{1 g}$. The non-bonding $E_{2 u}$ energy level is largely determined by the constituting linker E', orbitals, while the $\mathrm{B}_{1 \mathrm{~g}}$ orbital is determined by the interactions between metal $d$ and linker $A_{2}^{\prime \prime}$ orbitals. To relate $\mathrm{B}_{1 \mathrm{~g}}$ energy with atomic/molecular levels, we use a fiveorbital tight-binding model consisting of three metal $d$ orbitals and two linker $A_{2}^{\prime \prime}$ orbitals. The onsite energy of $A_{2}^{\prime \prime}$ is set to zero, so the onsite energy of $d$ orbitals are $E_{d}-A_{2}^{\prime \prime}$. Considering the periodic MOF networks of interest, each $A_{2}^{\prime \prime}$ couples with three $d$ orbitals, and each $d$ orbital couples with two $A_{2}^{\prime \prime}$ orbitals with coupling strength $t_{\pi d}$. Assuming no interaction among $d$ orbitals or among $A_{2}^{\prime \prime}$ orbitals, the resulting tight-binding Hamiltonian at $\Gamma$-point is

$$
H=\left(\begin{array}{ccccc}
0 & 0 & -t_{\pi d} & -t_{\pi d} & -t_{\pi d} \\
0 & 0 & -t_{\pi d} & -t_{\pi d} & -t_{\pi d} \\
-t_{\pi d} & -t_{\pi d} & E_{d-A_{2}^{\prime \prime}} & 0 & 0 \\
-t_{\pi d} & -t_{\pi d} & 0 & E_{d-A_{2}^{\prime \prime}} & 0 \\
-t_{\pi d} & -t_{\pi d} & 0 & 0 & E_{d-A_{2}^{\prime \prime}}
\end{array}\right)
$$

The spectra of this Hamiltonian include a linker-orbital only state

$$
E_{1}=0
$$

doubly degenerate metal d-orbital only states

$$
E_{2}=E_{3}=E_{d-A_{2}^{\prime \prime}}
$$


a bonding state

$$
E_{4}=\frac{\Delta E_{d-A_{2}^{\prime \prime}}}{2}-\frac{1}{2} \sqrt{\Delta E_{d}^{2}-A_{2}^{\prime \prime}+24 t_{\pi d}^{2}}
$$

and finally, an anti-bonding state that corresponds to the $\mathrm{B}_{1 \mathrm{~g}}$ state

$$
E_{5}=\frac{\Delta E_{d-A_{2}^{\prime \prime}}}{2}+\frac{1}{2} \sqrt{\Delta E_{d}^{2}-A_{2}^{\prime \prime}+24 t_{\pi d}^{2}}
$$

By fitting the first principles values with the energy level $E_{5}$ together with the energies of linkeronly and metal-only states, we obtained the parameters given in Table S1.

\section{Further details on first principles calculations}

The first principles electronic structures were calculated on a $4 \times 4 \times 1 \boldsymbol{k}$-grid using QUANTUM ESPRESSO ${ }^{14,15}$. Norm-conserving pseudopotentials from PseudoDojo ${ }^{16}$ were employed with a kinetic energy cut-off of $100 \mathrm{Ry}$. Vacuum spacing larger than $10 \AA$ and Coulomb cut-off technique ${ }^{17}$ were employed together to avoid the spurious interactions between image layers in the periodic boundary condition. We used the same parameters for both structure relaxation and band structure calculations. To improve the commonly underestimated band gap prediction by local or semi-local density functionals, we benchmarked the Ni-BHT band gap predictions using generalized gradient approximation (GGA) functional, hybrid functional, and GGA with Hubbard $U$ correction, using the result with $G W$ self-energy correction ${ }^{18}$ as the reference. The GGA functional of Perdew, Burke, and Ernzerhof $(\mathrm{PBE})^{19}$, and corresponding hybrid functionals with bare $(\mathrm{PBE} 0)^{20}$ and screened (HSE06) ${ }^{21,22}$ exchange terms are employed. Different effective Hubbard $U$ values of $4 \mathrm{eV}$ and $6 \mathrm{eV}$ are also tested in combination with PBE. The $G W$ quasiparticle band was calculated using BerkeleyGW ${ }^{18} .30$ Ry and 60 Ry cut-off energies were used for screened and bare Coulomb interactions. Over 1000 empty bands were used to reach convergence. By comparing with the reliable but expensive $G W$ calculation as the reference, we found that PBE0 performed the best in terms of band gap prediction, while all other approximations significantly underestimate the band gap of Ni-BHT, as shown in Figure S4. Therefore, we employ PBE0 for all electronic structure calculations in this work. Wannier interpolations were performed using Wannier $90^{23,24}$ program starting from the same $\boldsymbol{k}$-grid. Then the Wannier-basis Hamiltonians were fed into Wanniertools ${ }^{25}$ to simulate the edge states and to calculate the $Z_{2}$ numbers. 
$\mathrm{Ab}$ initio molecular dynamics simulations were carried out for a Pd-HIB supercell with 468 atoms and $20 \AA$ vacuum spacing to avoid finite size effect. Calculations were performed using Vienna $\mathrm{Ab}$ initio Simulation Package (VASP) with $300 \mathrm{eV}$ cutoff energy, 1.5 fs time step, $10^{-4} \mathrm{eV}$ electronic self-consistency criterion, and NVT ensemble using Nosé-Hoover thermostat. The simulated system temperature and energy, as well as final structure after the AIMD simulations, are shown in Figure SX. The stably oscillating temperature, energy and structure demonstrate that the Pd-HIB system can be thermally stable at least up to this temperature. 


\section{Supplementary References}

(1) Sheberla, D.; Bachman, J. C.; Elias, J. S.; Sun, C. J.; Shao-Horn, Y.; Dincă, M. Conductive MOF Electrodes for Stable Supercapacitors with High Areal Capacitance. Nat. Mater. 2017, 16, 220-224.

(2) Wu, G.; Huang, J.; Zang, Y.; He, J.; Xu, G. Porous Field-Effect Transistors Based on a Semiconductive Metal-Organic Framework. J. Am. Chem. Soc. 2017, 139, 1360-1363.

(3) Wang, B.; Luo, Y.; Liu, B.; Duan, G. Field-Effect Transistor Based on an in Situ Grown Metal-Organic Framework Film as a Liquid-Gated Sensing Device. ACS Appl. Mater. Interfaces 2019, 11, 35935-35940.

(4) Zhou, Q.; Wang, J.; Chwee, T. S.; Wu, G.; Wang, X.; Ye, Q.; Xu, J.; Yang, S. W. Topological Insulators Based on 2D Shape-Persistent Organic Ligand Complexes. Nanoscale 2015, 7, 727-735.

(5) Silveira, O. J.; Chacham, H. Electronic and Spin-Orbit Properties of the Kagome MOF Family $\mathrm{M}_{3}(1,2,5,6,9,10 \text {-Triphenylenehexathiol })_{2}(\mathrm{M}=\mathrm{Ni}, \mathrm{Pt}, \mathrm{Cu}$ and $\mathrm{Au}) . J$. Phys. Condens. Matter 2017, 29, 09LT01.

(6) Huang, X.; Sheng, P.; Tu, Z.; Zhang, F.; Wang, J.; Geng, H.; Zou, Y.; Di, C.; Yi, Y.; Sun, Y.; et al. A Two-Dimensional $\pi-d$ Conjugated Coordination Polymer with Extremely High Electrical Conductivity and Ambipolar Transport Behaviour. Nat. Commun. 2015, 6, 7408.

(7) Zhang, X.; Zhou, Y.; Cui, B.; Zhao, M.; Liu, F. Theoretical Discovery of a Superconducting Two-Dimensional Metal-Organic Framework. Nano Lett. 2017, 17, 6166-6170.

(8) Huang, X.; Zhang, S.; Liu, L.; Yu, L.; Chen, G.; Xu, W.; Zhu, D. Superconductivity in a Copper(II)-Based Coordination Polymer with Perfect Kagome Structure. Angew. Chemie Int. Ed. 2018, 57, 146-150.

(9) Shi, W.; Wu, G.; Yong, X.; Deng, T.; Wang, J.-S.; Zheng, J.-C.; Xu, J.; Sullivan, M. B.; Yang, S.-W. Orbital-Engineering-Based Screening of $\pi$-Conjugated d 8 Transition-Metal Coordination Polymers for High-Performance n-Type Thermoelectric Applications. ACS Appl. Mater. Interfaces 2018, 10, 35306-35315.

(10) Yong, X.; Shi, W.; Wu, G.; Goh, S. S.; Bai, S.; Xu, J.-W.; Wang, J.-S.; Yang, S.-W. Tuning the Thermoelectric Performance of $\pi-\mathrm{d}$ Conjugated Nickel Coordination Polymers through Metal-Ligand Frontier Molecular Orbital Alignment. J. Mater. Chem. A 2018, 6, 19757-19766.

(11) Li, S.; Lü, T.-Y.; Zheng, J.-C.; Yang, S.-W.; Wang, J.-S.; Wu, G. Origin of Metallicity in 2D Multilayer Nickel Bis(Dithiolene) Sheets. 2D Mater. 2018, 5, 035027.

(12) Shi, W.; Wu, G.; Hippalgaonkar, K.; Wang, J.-S.; Xu, J.; Yang, S.-W. Poly(NickelEthylenetetrathiolate) and Its Analogs: Theoretical Prediction of High-Performance Doping-Free Thermoelectric Polymers. J. Am. Chem. Soc. 2018, 140, 13200-13204.

(13) Shi, W.; Wong, Z. M.; Deng, T.; Wu, G.; Yang, S. Unravelling the Molecular Origin of 
Organic Semiconductors with High-Performance Thermoelectric Response. Adv. Funct. Mater. 2021, 31, 2007438.

(14) Giannozzi, P.; Baroni, S.; Bonini, N.; Calandra, M.; Car, R.; Cavazzoni, C.; Ceresoli, D.; Chiarotti, G. L.; Cococcioni, M.; Dabo, I.; et al. QUANTUM ESPRESSO: A Modular and Open-Source Software Project for Quantum Simulations of Materials. J. Phys. Condens. Matter 2009, 21, 395502.

(15) Giannozzi, P.; Andreussi, O.; Brumme, T.; Bunau, O.; Buongiorno Nardelli, M.; Calandra, M.; Car, R.; Cavazzoni, C.; Ceresoli, D.; Cococcioni, M.; et al. Advanced Capabilities for Materials Modelling with Quantum ESPRESSO. J. Phys. Condens. Matter 2017, 29, 465901.

(16) van Setten, M. J.; Giantomassi, M.; Bousquet, E.; Verstraete, M. J.; Hamann, D. R.; Gonze, X.; Rignanese, G.-M. The PseudoDojo: Training and Grading a 85 Element Optimized Norm-Conserving Pseudopotential Table. Comput. Phys. Commun. 2018, 226, $39-54$.

(17) Sohier, T.; Calandra, M.; Mauri, F. Density Functional Perturbation Theory for Gated Two-Dimensional Heterostructures: Theoretical Developments and Application to Flexural Phonons in Graphene. Phys. Rev. B 2017, 96, 075448.

(18) Deslippe, J.; Samsonidze, G.; Strubbe, D. A.; Jain, M.; Cohen, M. L.; Louie, S. G. BerkeleyGW: A Massively Parallel Computer Package for the Calculation of the Quasiparticle and Optical Properties of Materials and Nanostructures. Comput. Phys. Commun. 2012, 183, 1269-1289.

(19) Perdew, J. P.; Burke, K.; Ernzerhof, M. Generalized Gradient Approximation Made Simple. Phys. Rev. Lett. 1996, 77, 3865-3868.

(20) Perdew, J. P.; Ernzerhof, M.; Burke, K. Rationale for Mixing Exact Exchange with Density Functional Approximations. J. Chem. Phys. 1996, 105, 9982-9985.

(21) Heyd, J.; Scuseria, G. E.; Ernzerhof, M. Hybrid Functionals Based on a Screened Coulomb Potential. J. Chem. Phys. 2003, 118, 8207-8215.

(22) Krukau, A. V.; Vydrov, O. A.; Izmaylov, A. F.; Scuseria, G. E. Influence of the Exchange Screening Parameter on the Performance of Screened Hybrid Functionals. J. Chem. Phys. 2006, 125, 224106.

(23) Mostofi, A. A.; Yates, J. R.; Lee, Y.-S.; Souza, I.; Vanderbilt, D.; Marzari, N. Wannier90: A Tool for Obtaining Maximally-Localised Wannier Functions. Comput. Phys. Commun. 2008, 178, 685-699.

(24) Pizzi, G.; Vitale, V.; Arita, R.; Blügel, S.; Freimuth, F.; Géranton, G.; Gibertini, M.; Gresch, D.; Johnson, C.; Koretsune, T.; et al. Wannier90 as a Community Code: New Features and Applications. J. Phys. Condens. Matter 2020, 32, 165902.

(25) Wu, Q. S.; Zhang, S. N.; Song, H. F.; Troyer, M.; Soluyanov, A. A. WannierTools: An Open-Source Software Package for Novel Topological Materials. Comput. Phys.

Commun. 2018, 224, 405-416. 